\title{
Changes of Permutation Pattern Entropy and Ordinal Pattern Entropy during Three Emotion States: Natural, Happiness and Sadness
}

\author{
Yirong Xia ${ }^{1}$, Licai Yang ${ }^{1}$, Hongyu Shi ${ }^{1}$, Yuan Zhuang $^{1}$, Chengyu Liu ${ }^{2}$ \\ 1. School of Control Science and Engineering, Shandong University, Jinan, China \\ 2. School of Instrument Science and Engineering, Southeast University, Nanjing, China
}

\begin{abstract}
Analysis of heart rate variability (HRV) holds an important potential in emotion recognition, especially when the non-linear entropy methods are involved. Permutation pattern entropy (PPEn) and ordinal pattern entropy (OPEn) are two important Shannon entropy approaches for analyzing the inherent dynamic characteristics of RR interval time series and their performances should be tested in the emotion study.

In this study, ECG signals were recorded from 60 healthy subjects by RM6240B multi-channel signal acquirement system under three emotion states: natural, happiness and sadness, at a sample rate of $1000 \mathrm{~Hz}$. For each emotion state, ECG signal was recorded for five minutes. $Q R S$ complexes were identified and then the RR interval time series were analyzed by the PPEn and OPEn methods.

Compared with natural emotion (1.6834 \pm 0.06$)$, PPEn increases significantly in both happiness $(1.7205 \pm 0.05)$ and sadness (1.7065 \pm 0.05$)$ emotions. Meanwhile, PPEn shows significant difference between happiness and sadness emotions. For OPEn, compared with natural emotion (1.8318 \pm 0.07$)$, it also increases in both

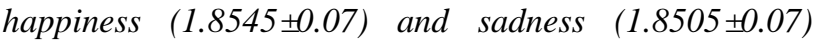
emotions. However, there is no significant difference between happiness and sadness emotions.

The changes of PPEn and OPEn values during the three different emotion states suggests that emotion has a non-negligible influence on the inherent dynamic characteristics in cardiovascular system, and the PPEn and OPEn methods can be used in the emotion recognition study.
\end{abstract}

\section{Introduction}

Heart rate variability (HRV) indices for emotion recognition have received great attention. As heart rate regulation is a complex phenomenon [1], some nonlinear indices have been employed in emotion recognition. Yang et al. [2] found that the index, SD1 in Poincare plots increased significantly for fear comparing with calmness state, and Goshvarpour et al. [3] reported that the poincare plots of RRI time series are cigar-shaped in relaxation emotional state, while round cloud of points during happiness, sadness and fear emotional states. The dominant lyapunov exponent was extracted from RRI time series and an overall accuracy of $79.29 \%$ was achieved in recognizing four emotional states (happiness, sadness, anger and relaxation), with $79.15 \%$ on the valence axis and $83.55 \%$ on the arousal axis [4]. Valenza et al. found that the median approximate entropy decreases during arousal elicitation with neutral elicitation. Shi et al. has reported that there was significant difference of fuzzy measure entropy between happiness and sadness emotional states [5].

Among the nonlinear approaches, permutation patterns entropy (PPEn) and ordinal patterns entropy (OPEn) play an important role and they have been used to characterize RRI time series in different fields, respectively. Bandt and Pompe introduced the concept of PPEn from Shannon entropy in 2002 [6]. Graff et al. [7] reported that PPEn was significantly different between groups with cardio depressive or negative results for the head-up tilt test. Taherkhani et al. found that the fluctuation value for the permutation entropy of a heart failure person was higher than a healthy person [8]. PPEn of RRI time series can be observed that the mean value due to non-shockable ventricular arrhythmia class was higher than shockable VA class [9]. Then, the OPEn follows the PPEn was considered to deal with the equals of segments [10-12]. Bian et al. presented that OPEn could effectively distinguish young and elderly group from RRI time series [10]. Makowiec et al. showed that OPEn grew systematically with age and it increased depended on gender [11]. Wejer et al. [12] confirmed that OPEn reduced after the tilt compared to at rest for both vasovagal patients and healthy people groups [12].

However, little attention has been paid so far to PPEn and OPEn of RRI time series for emotion recognition. Thus, this study is aimed to quantify how the PPEn and OPEn of RRI time series change among natural, happiness and sadness emotional states. 


\section{Method}

\subsection{Subjects}

The group consisted of 60 healthy subjects with a mean age of 23 years ( 30 females and 30 males). All subjects signed the consent forms in the study. The details of subjects are summarized as male, female and total in Table 1.

Table 1. Basic clinical characteristics from all 60 subjects.

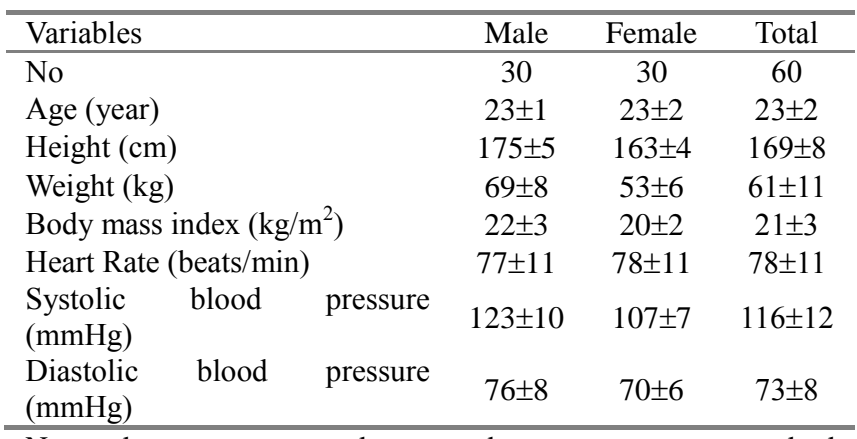

Note: data are expressed as numbers or mean \pm standard deviation (SD).

\subsection{Experimental procedure and data collection}

For each subject, a five minutes ECG signal was recorded at a rate of $1000 \mathrm{~Hz}$ by a RM6240B signal acquirement system during the natural, happiness and sadness emotional states. Video clips of 'World Heritage In China' (a documentary), 'Top Funny Comedian' (a situation comedy) and 'Nuan Chun' (a touching movie) were used to induce natural, happiness and sadness emotional states, respectively. Before replaying the emotion-stimulation clips, 10 minutes were given to the subjects to calm themselves down. Then emotionstimulation videos were replayed to the subjects with randomized order. A gap of 10 minutes between each two videos replaying was used to make sure subjects could restore from the previous emotional state.

Then, $\mathrm{R}$ peaks were detected by the difference threshold algorithm [13] after using a sym8 wavelet filter to remove the high-frequency interference and baseline drift in ECG signal [14]. Additionally, we checked each ECG signal and manually removed the error $\mathrm{R}$ peaks before generating the RRI time series. The length of RRI time series is about 300 to 400 intervals for each emotional state of each subject.

\subsection{Permutation entropy and ordinal patterns entropy}

The Shannon entropy is an important index of describing complex phenomenon. It is defined as:

$$
S(X)=-\sum_{i} p\left(x_{i}\right) \ln p\left(x_{i}\right)
$$

where $p\left(x_{i}\right)$ is the corresponding probability to the observed value $x_{i}$ among the signal $X$.

Permutation entropy is the Shannon entropy of the distribution of permutation patterns initially introduced by Bandt and Pompe [6]. In order to create permutation patterns, two parameters, the embedding dimension $D>1$ ( $D \in N$, number of elements to be compared with each other from time series) and embedding delay $\tau$ ( $\tau \in N$, time separation between elements) are chosen. For the embedding dimension $D$, it relies on the length $N$ of time series under a certain circumstance $N>>D$ !, in addition, $D$ ! patterns can be obtained from the time series.

Supposing $D=3$, there will be six permutation patterns $\pi$ as shown in Figure 1 .

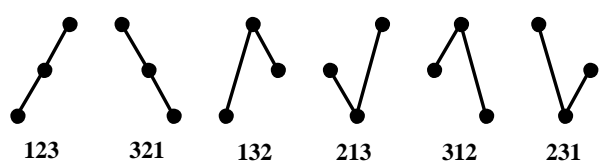

Figure 1. The six permutation patterns when embedding dimension $D=3$.

The above definitions apply to continuous time series so that equal values are very rare. If the values in a segment are the same, it is proposed to arrange these values by adding each of them a small random value to establish the order generally.

In order to process equals, specially, ordinal patterns depend on permutation patterns were defined. Supposing $D=3$, thirteen distinct ordinal patterns are shown in figure 2. Ordinal pattern entropy is calculated as the Shannon entropy with ordinal patterns distribution.

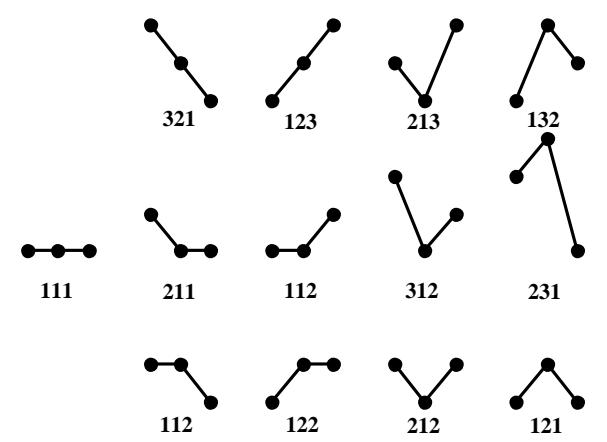

Figure 2. The thirteen ordinal patterns when embedding dimension $D=3$.

Owing to the length of RRI time series (300-400 data points), embedding dimension $D=3$ and embedding delay $\tau=1$ of PPEn and OPEn have been considered in this paper.

\subsection{Statistical analysis}


Normal distributions of PPEn and OPEn were tested by the Kolmogorov-Smirnov test. The results presented that data were normally distributed. Hence, the differences between the three emotional states (natural, happiness and sadness) were compared by paired $t$-Student test. In addition, the effect of gender factor on PPEn and OPEn results was also tested. All statistical analyses were performed using the SPSS software (Ver. 20, IBM, USA). The statistical significance was accepted at $P<0.05$.

\section{Results}

\subsection{Comparisons of permutation patterns entropy and ordinal pattern entropy}

The statistical results (mean and SD) of PPEn is shown in Figure 3, compared with natural emotional state (1.6834 \pm 0.06$)$, PPEn increases significantly in both happiness $\quad(1.7205 \pm 0.05, \quad P<0.01) \quad$ and sadness $(1.7065 \pm 0.05, \quad P<0.01)$ emotional states. Meanwhile, PPEn has significant difference $(\mathrm{P}<0.05)$ between happiness and sadness emotional states.

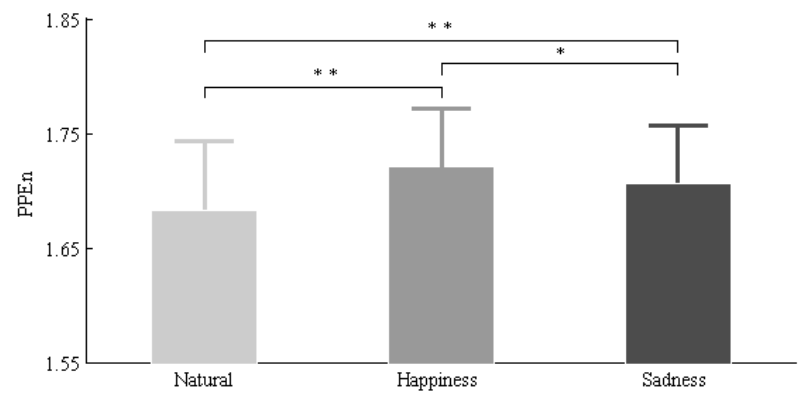

Figure 3. Mean and SD of PPEn among the three emotional states. ** means $\mathrm{P}<0.01$, * means $\mathrm{P}<0.05$.

As seen Figure 4, OPEn increases both in happiness $(1.8545 \pm 0.07, P<0.05)$ and sadness $(1.8505 \pm 0.07, \mathrm{P}<0.01)$ emotional states compared with natural emotional state (1.8318 \pm 0.07$)$. However, there was no significant difference $(P \geq 0.05)$ between happiness and sadness emotional states.

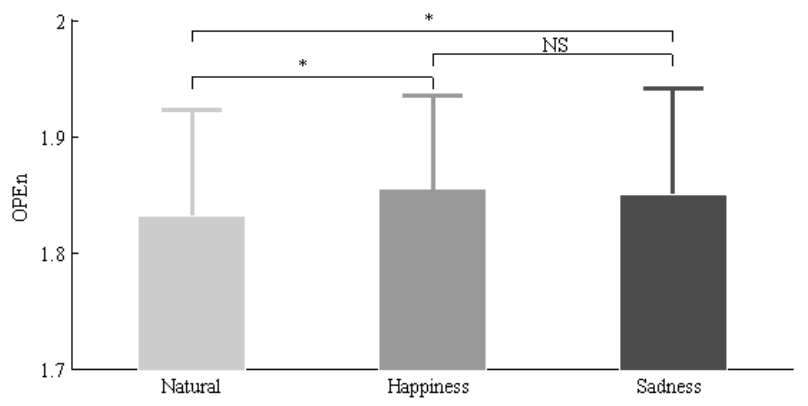

Figure 4. Mean and SD of OPEn among the three emotional states. * means $\mathrm{P}<0.05$, NS means $\mathrm{P} \geq 0.05$.

\subsection{Comparisons of genders}

Since the enrolled subjects had the same amount of females and males in this study (30 males and 30 females), the effect of gender factor of PPEn and OPEn for three emotion states was tested. As shown in Table 2, group-t test results showed that there was no significant difference between females and males in both PPEn and OPEn for the three emotion states.

Table 2. Effect of gender factor on pattern entropies for three emotion states.

\begin{tabular}{ccccc}
\hline \multirow{2}{*}{ Index } & \multirow{2}{*}{ Emotion } & \multicolumn{2}{c}{ Gender } & \multirow{2}{*}{$P$} \\
\cline { 3 - 5 } PPEn & Natural & $1.6803 \pm 0.06$ & $1.6866 \pm 0.07$ & 0.70 \\
& Happiness & $1.7177 \pm 0.06$ & $1.7232 \pm 0.05$ & 0.70 \\
& Sadness & $1.7120 \pm 0.04$ & $1.7010 \pm 0.06$ & 0.40 \\
OPEn & Natural & $1.8321 \pm 0.07$ & $1.8315 \pm 0.07$ & 0.98 \\
& Happiness & $1.8504 \pm 0.06$ & $1.8585 \pm 0.08$ & 0.66 \\
& Sadness & $1.8628 \pm 0.07$ & $1.8383 \pm 0.07$ & 0.18 \\
\hline
\end{tabular}

Note: data are expressed as numbers or mean \pm standard deviation (SD).

\section{Conclusion}

This study aimed at characterizing the dynamics of RRI time series by using two nonlinear indices: PPEn and OPEn during natural, happiness and sadness emotional states. The results showed that PPEn and OPEn increased significant both in happiness and sadness emotional states compared with natural emotional state. The results presented that permutation and ordinal pattern distribution of RRI time series are different between natural and non-natural (happiness and sadness) emotional states. It means that RRI time series of nonnatural emotional states become more complex. In addition, PPEn increased significant in happiness emotional state compared with sadness emotional state, but no difference for OPEn. In conclusion, this study could help to better understand the differences between the RRI time series of different emotional states.

\section{Acknowledgements}

This research was sponsored by the Natural Science Foundation of Shandong Province in China (grant 2014ZRE27230), the Key Research and Development Program of Shandong Province (grant 2016GSF120009) and the National Natural Science Foundation of China (grants 61671275 and 61201049), and the project of Southeast-Lenovo wearable Heart-Sleep-Emotion intelligent monitoring Lab.

\section{References}


[1] Voss A, Schulz S, Schroeder R, Baumert M, Caminal P. Methods derived from nonlinear dynamics for analysing heart rate variability. Philosophical Transactions of the Royal Society A-Mathematical Physical and Engineering Sciences. 2009;367(1887):277-96.

[2] Yang M, Liu G, Wen W. Nonlinear analysis of ECG and HRV signals with two types of emotional states. Journal of Computer Applications. 2012;32(10):2963-76.

[3] Goshvarpour A, Abbasi A, Goshvarpour A. Indices from lagged poincare plots of heart rate variability: an efficient nonlinear tool for emotion discrimination. Australasian Physical \& Engineering Sciences in Medicine. 2017.

[4] Valenza G, Allegrini P, Lanata A, Scilingo EP. Dominant Lyapunov exponent and approximate entropy in heart rate variability during emotional visual elicitation. Frontiers in Neuroengineering. 2012;5(3):1-7.

[5] Shi H, Yang L, Zhao L, Su Z, Mao X, Zhang L et al. Differences of heart rate variability between happiness and sadness emotion states: a pilot study. Journal of Medical \& Biological Engineering. 2017;4(37):527-39.

[6] Bandt C, Pompe B. Permutation entropy: a natural complexity measure for time series. Physical Review Letters. 2002;88(17):174102.

[7] Graff B, Graff G, Makowiec D, Kaczkowska A, Wejer D, Budrejko $\mathrm{S}$ et al. Entropy measures in the assessment of heart rate variability in patients with cardiodepressive vasovagal syncope. Entropy. 2015;17(3):1007-22.

[8] Taherkhani F, Rahmani M, Taherkhani F, Akbarzadeh H, Abroshan H. Permutation entropy and detrend fluctuation analysis for the natural complexity of cardiac heart interbeat signals. Physica A: Statistical Mechanics and its Applications. 2013;392(14):3106-12.

[9] Tripathy RK, Sharma LN, Dandapat S. Detection of shockable ventricular arrhythmia using variational mode decomposition. Journal of Medical Systems. 2016;40(4):79.
[10] Bian C, Qin C, Ma Q, Shen Q. Modified permutationentropy analysis of heartbeat dynamics. Physical Review E. 2012;85(2):021906.

[11] Makowiec D, Kaczkowska A, Wejer D, ZarczynskaBuchowiecka M, Struzik ZR. Entropic measures of complexity of short-term dynamics of nocturnal heartbeats in an aging population. Entropy. 2015;17(3):1253-72.

[12] Wejer D, Graff B, Makowiec D, Budrejko S, Struzik ZR. Complexity of cardiovascular rhythms during head-up tilt test by entropy of patterns. Physiological Measurement. 2017;38(5):819-32.

[13] Pan J, Tompkins WJ. A real-time QRS detection algorithm. IEEE Transactions on Biomedical Engineering. 1985;32(3):230-6.

[14] Rioul O, Vetterli M. Wavelets and signal processing. IEEE Signal Processing Magazine. 1991;8(4):14-38.

Address for correspondence.

Licai Yang (yanglc@sdu.edu.cn)

School of Control Science and Engineering

Shandong University, Jinan, China

or

Chengyu Liu (bestlcy@sdu.edu.cn)

School of Instrument Science and Engineering

Southeast University, Nanjing, China 